\title{
Energy Price-Based Control Strategy of a Small-Scale Head-Dependent Hydroelectric Power Plant
}

\author{
R. Tiainen, T. Lindh, J. Ahola, M. Niemelä and V. Särkimäki \\ Department of Electrical Engineering \\ Lappeenranta University of Technology \\ P.O. Box 20, 53851 Lappeenranta (Finland) \\ Phone: +358 56216772 , Fax: +358 56216799 , \\ E-mail: risto.tiainen@lut.fi, tuomo.lindh@lut.fi,jero.ahola@lut.fi,markku.niemela@lut.fi, ville.sarkimaki@lut.fi
}

\begin{abstract}
In deregulated electricity markets, the market price of electricity can be used to control power generation in order to obtain maximum revenue. This makes the building of smaller and smaller hydroelectric power stations economically feasible. High price of energy indicates that power demand is high relative to production capacity. Therefore, price-based optimization will enable the production of electricity when it is most needed. In this article, the feasibility of market price based control strategy for a small-scale hydroelectric power station is evaluated. An actual Finnish small-scale hydroelectric power plant is used as the basis for the modeling.
\end{abstract}

\section{Key words}

Distributed generation, electricity market, hydroelectric power generation, optimization.

\section{Introduction}

The liberalization of electricity markets in the Nordic countries (Denmark, Finland, Norway, and Sweden) began in the early 1990's. The first international power exchange, Nordpool, was opened in 1996. Later, the dayahead physical spot market (Elspot) trade was transferred to a separate company, Nord Pool Spot [1]. Elspot contracts are traded daily for delivery during the next 24 hours [2][3]. The spot prices are publicly available free of charge on the Nord Pool Spot web page after the trading for a given day has ended [4]. To obtain the pricing data during trading, commercial services provided by Nord Pool Spot must be used. If a small-scale energy producer is credited by the retailer based on hourly metering of the amount of energy generated and the corresponding spot price, the producer can optimize the generation to obtain maximum revenue. High price of energy is generally an indication of high demand of energy in proportion to production. Therefore, with price-based optimization, the water in the reservoir can be utilized when it is most needed.

Hydroelectric power production is characterized by high investment costs compared to operational costs. The expected repayment time often defines whether it is economically feasible to establish a power station in a given location. In small-scale hydroelectric plants, the reservoirs are typically small in volume, and the energy production affects significantly the water level in the reservoir. If this is the case, energy should be produced when it generates the most revenue. If the available energy can be utilized more effectively in terms of generated revenue, smaller and smaller hydroelectric power plants will become feasible to establish

In this paper, we investigate the optimization of the energy production of a Finnish small-scale hydroelectric power plant based on the Nord Pool Spot hourly energy price. First, the characteristics of the energy price and obtaining the price at the power plant are discussed. Second, the installation that is used as a basis for the optimization model and simulations is described. Third, the plant model and the optimization algorithm are described. Finally, simulation results are presented and conclusions drawn.

\section{The Energy Price}

\section{A. Obtaining the Energy Price}

As previously discussed, the Elspot price is publicly available on the Nord Pool spot web site. From there, it must be transferred to the power station. Typically, distributed generation involves communications for different purposes, such as energy metering, protection, production supervision, condition monitoring etc. Communication systems and media used can be, e.g., public mobile telephone networks (GSM in Europe), private radio networks, satellite communications, or the public telephone network. In addition, broadband Internet access is increasingly often available in rural environment, where hydroelectric power stations are typically located. These can be provided using, e.g., some variant of DSL (Digital Subscriber Line), which uses telephone lines as a communication channel. In the future, wireless technologies, such as $3 \mathrm{G}$ mobile telephone technologies and WiMAX, can extend 
broadband Internet access to areas that do not have wired telephone networks.

\section{B. Characteristics of the Energy Price}

The spot energy price can vary significantly within a day and between consecutive days. Long time trends that are caused by e.g. the amount of rainfall and weatherdependent consumption changes (e.g., increased need of heating during extreme cold) span so long time periods compared to the optimization window that they do not directly affect the optimization in the case of small-scale head-dependent plants.

During workdays, the spot electricity price is typically lower in the night than during work-hours. In the weekends the price is steadier. In order to determine the value of the water in the reservoir at the end of the optimization period, the optimization algorithm requires the knowledge of the energy price for some time after the end of the period. This is due to the fact that the value of the reservoir content is determined by the revenue that can be generated from it, and the generators determine the maximum power that can be utilized to produce the energy. Fig. 1 gives an example of the Elspot price on a Friday and the following Saturday (data acquired from the Elspot web site [4]). During the Friday, the highest Elspot price (from 11 to 12) is approximately twice as high as the lowest one (from 3 to 4), whereas the Saturday's prices change very little. The energy price can be predicted to some accuracy by assuming that the next day's prices will be the same as todays, unless the transition is from a working day to weekend or viceversa, in which case the previous similar day can be used as a guess.

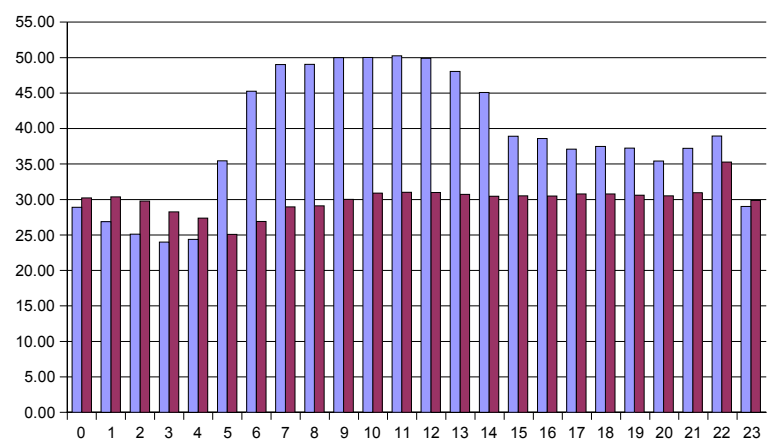

Fig. 1. Elspot price in Euros/MWh for each hour on Friday, September 12, 2005 (columns on the left) and Saturday, September 13, 2005 (columns on the right).

\section{Pilot Power Plant}

\section{A. Generators and Control}

The pilot power station is situated in Tirva, a small village near Lappeenranta in South-Eastern Finland. The pilot plant consists of four turbogenerator units. All units are equipped with fixed blade turbines. Four of the generators are directly grid connected, and one is driven by a four-quadrant frequency converter. The frequency range of the variable speed generator is 0.6-1.2 times the nominal frequency $(50 \mathrm{~Hz})$, and the nominal power of the variable speed generator is $50 \mathrm{~kW}$. For a nominal head this turbine gives $30-60 \mathrm{~kW}$ using the full frequency range. The nominal powers of the other generators are 20 $\mathrm{kW}, 30 \mathrm{~kW}$, and $100 \mathrm{~kW}$. The power is controlled by switching the generators on and off, and controlling the frequency of the converter-driven generator. In this research, the efficiencies of generators are assumed equal, and the losses in the frequency converter are neglected.

\section{B. Communications}

At the pilot installation site, two telephone lines were available. One is used for energy metering, whereas the other was not used. An ADSL Internet connection was available and was acquired using the previously unused telephone line. Thus the ADSL modem should not be able to disturb energy metering. The energy price is fetched from the Nord Pool Spot WWW page for each day the previous evening.

\section{Optimization Principle}

The objective of the optimization is to maximize the income in a given time window, 1-2 days in this case, with the hourly-changing energy price, the inflow, and the current generator states as inputs while keeping the head between required limits. The value of the pool at the end of the optimization window must be taken into account in the optimization. The value is a function of the head at the end of the optimization window, the energy prices of the next optimization window, and the inflow. A small-scale power producer is a price taker, i.e., the power production has no impact on the electricity price. Generally, power production scheduling can be divided into long-term scheduling (years, granularity of a week), medium-term scheduling (up to a year, the same granularity as in long-term model), and short-term scheduling (up to days, granularity of an hour) [5]. In the case of a small-scale hydroelectric power production, the volume of the reservoir is so small that long or mediumterm scheduling is not required; the level of the water in the reservoir can be driven to any value between the limits in a matter of hours or days.

\section{A. The Objective Function}

The optimization problem can be stated as (based on the formulations of Conejo et al. [6] and Mendez et al. [7]), maximize

$J=V_{H}+\Delta \mathrm{T} \sum_{\mathrm{i}=1}^{\mathrm{K}}\left(\pi_{\mathrm{i}} \sum_{\mathrm{j}=1}^{\mathrm{G}} p_{\mathrm{ij}}\right)-\sum_{\mathrm{i}=1}^{\mathrm{K}} \sum_{\mathrm{j}=1}^{\mathrm{G}} \pi_{\mathrm{sw}, \mathrm{j}} s w_{\mathrm{ij}}$,

where the symbols are

$V_{\mathrm{H}} \quad$ value of the water in the pool at the end of the optimization window,

$\Delta \mathrm{T} \quad$ time granularity (1 hour),

$\mathrm{K}$ length of the optimization window (e.g. 24 hours), 
$p_{\mathrm{ij}} \quad$ power of generator $\mathrm{j}$ at hour $\mathrm{i}$ (the maximum power per generator is head-dependent),

$\pi_{\mathrm{i}} \quad$ electricity price at hour $\mathrm{i}$,

$\pi_{\mathrm{sw}, \mathrm{j}} \quad$ switching cost of generator $\mathrm{j}$,

$s w_{\mathrm{ij}} \quad$ number of off-switchings of generator $\mathrm{j}$ at hour i $(0$ or 1$)$.

Furthermore, the water balance equation is valid,

$q_{\text {in }}=q_{0}+\sum_{\mathrm{i}=1}^{\mathrm{G}} q_{\mathrm{i}}+\frac{\mathrm{d} V}{\mathrm{~d} t}$,

where $q_{\text {in }}$ is the incoming flow $\left(\mathrm{m}^{3} / \mathrm{s}\right)$ of the reservoir, $q_{0}$ is the spillage, $q_{\mathrm{i}}(\mathrm{i} \geq 1)$ are the flows of the individual generators, and $V$ is the volume of the reservoir. Hydrological constraints may include, e.g., minimum discharge or minimum/maximum head.

The objective function, predicted income $J$, consists of three parts. The first, $V_{\mathrm{H}}$, is the value of the water in the reservoir at the end of the optimization window. This is a function of the predicted price of electricity after the optimization window, the head at the end of the optimization window, as well as the incoming flow. When the next day's optimal utilization is first calculated, the electricity prices of the day after that are not known. Different techniques - such as time series models, simulation-based methods, and neural networks (see e.g. [8],[9] and [10]) - have been presented for predicting future prices. As depicted before, however, an excessively simple method was used in this study. The next day's prices were assumed to equal the prices of the previous similar day (similar in terms of working day/non-working day). The time required to drain the pool to the lower head limit was then calculated ( $n$ hours), and an estimate for the value of the pool was then obtained by calculating the amount of energy produced during this time and multiplying it by the average price during the first $n$ hours of the following day. The method does not calculate, how the water would be optimally discharged, but merely the income obtained by draining the pool to the minimum level as quickly as possible, starting at the end of the optimization window (at midnight). However, the calculation is very simple, and the value obtained bears a connection to the real system. The determination of $V_{\mathrm{H}}$ could be improved by calculating an optimal utilization of generators for the next optimization window (day) with different initial heads.

The second term represents the revenue generated. For each hour (or, more generally, a time step of length $\Delta \mathrm{T}$ ), the sum of generator output powers is calculated, this is multiplied by the hour's price and the time step length yielding the income. The output power of the generators is a function of the head. It was assumed that the output power is nominal with the nominal head and changes linearly (being zero with zero head). This is justified by the fact that potential energy of the reservoir is a linear function of the head.
The third term represents the switching costs. The directly grid-connected generators are connected to the grid and disconnected from it by the use of circuit breakers. It was assumed that each breaker has a maximum number of off-switchings it will sustain before having to be replaced. The cost of replacing a circuit breaker consists of the cost of the equipment (i.e., the new breaker) and the replacement work, and possibly the lost water (if there is spillage, $q_{0}>0$ in equation 2). Based on estimates for the replacement costs and the maximum number of off-switchings, an off-switching cost was calculated for each directly grid-connected generator. For the converter-driven generator the switching cost is zero. The switching costs represent in this model the operation costs of the hydroelectric power plant, which are typically small compared to the investment costs.

\section{B. Boundary Conditions in the Pilot Case}

The river on which the pilot power plant at Tirva is situated runs from a small lake to another. The pilot plant is the only power plant along its short course. Thus, in the optimization, problem, there are no couplings between power plants (the utilization of one generator stage affecting the input flow of another). Furthermore, since the river is very short, the hydrological constrains are relatively relaxed, and, in the model, there was no minimum discharge, for example. In Fig. 2, a photograph from the pilot plant is shown. As can be seen, there is a lot of water at the moment of the picture's taking, and the spillage is high.

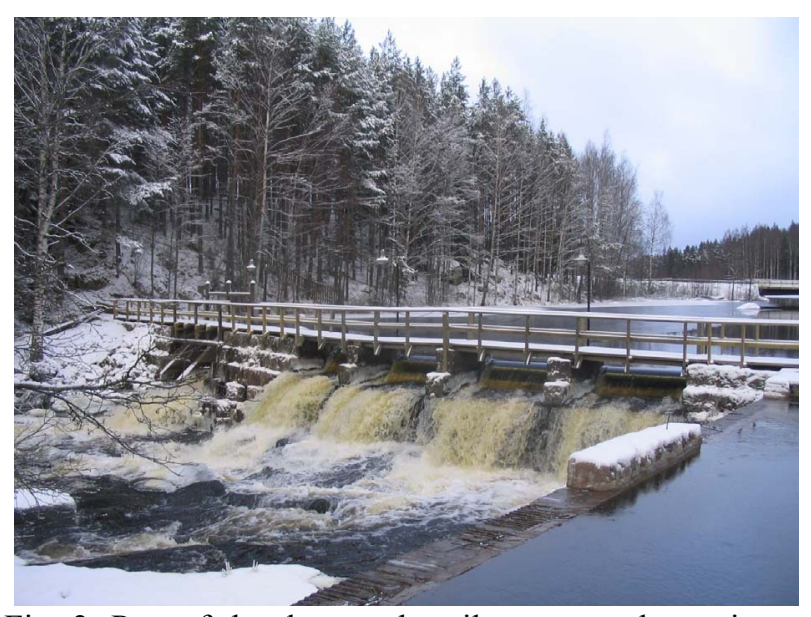

Fig. 2. Part of the dam at the pilot power plant, picture taken $29^{\text {th }}$ November 2007. The generator pool is on the right (partially visible). As can be seen, the hydrological situation is such that the inflow is greater than the plant's capacity, and the spillage is high.

For the simulation model, the water discharge through each of the generators has to be estimated. It was assumed that the flow remains essentially constant at nominal independent of head and, in the case of the frequency converter-driven generator, the rotation speed. These assumptions simplify the model. If greater accuracy is required, the constant relationships can be replaced with e.g. piecewise linear representations. When 
the discharge is assumed independent of the head and the output power is assumed linearly dependent on the head, running with high head is favored, since the amount of consumed water is constant and yet the power output is high when the head is high. In reality, the water discharge is not constant as a function of the head. The exact relationship is not known, but it can be assumed that the lower the head the less water will be discharged. This will make running the plant with low head slightly less disadvantageous than in the model with constant water discharge. In both cases, however, it can be qualitatively stated that it is generally feasible to store water in the pool when the price of the energy is low, and, conversely, discharge it when the price is high.

As previously discussed, due to the pilot plant being situated on a very short river between two lakes, there are no requirements for minimum discharge. However, the upper water storage is a lake, and its surface cannot be driven to any height (that is, the lake must not be drained). Therefore, the head has limitations that have to be observed. The upper limit arises from the height of the dam: water over this limit will be spilled. Lower limit is the minimum allowable height. In the case of the pilot plant, the nominal height is 5 meters. In the simulations, the upper limit used was 5.2 meters whereas the lower one was 3.5 meters. In practice, it would probably not be allowable to let the lake drain to 3.5 meters, and this limit would be higher. A low value was selected to allow greater set of possible generator utilization patterns for the optimizer in the simulations. However, due to the assumed constant discharge, it is very uneconomical to run the plant with a low head, and, therefore, the optimized generator utilization sequence was never such that this limit would be approached. Nonetheless, a virtual penalty was added to the objective function (Eq. 1) per each hour spent under the lower head limit to ensure the optimizer would not drain the pool.

\section{Optimization Algorithms}

The state of the generators on each hour was represented by a vector (enable, e) of four values. For the three directly grid-connected generators, their states could either be on (1) or off (0), whereas the converter-driven generator could be in the off-state $(0)$ or in the on-state with some range of normalized frequency. In the simulation, a range of 0.6-1.2 times the nominal rotation speed (and power) was used. The effect of the rotation speed to the efficiency of the turbine was not taken into account. The efficiency of a fixed blade angle turbine will drop when the rotation speed is significantly different from the nominal one [11]. If the shape of the turbine efficiency curve is known, the effect can be taken into account in the optimization. This kind of modeling results in the e vector having three binary integer values $(0 / 1)$ and one continuous value (0.6-1.2). A solution to the problem will comprise of e vectors for each hour of the day, yielding a vector of length $24 \cdot 4=96$ values, 24 of them continuous and the rest binary.
The formulation of the problem is such that numerical optimization methods appear most suitable for solving it. The mixed-integer nature of the representation of generator utilization may pose problems for some algorithms, though. This can be circumvented by, e.g., modeling the converter-driven generator as a set of virtual generators. For example, the $50 \mathrm{~kW}$ frequencyconverter driven generator (with assumed power range of 0.6...1.2 times nominal, corresponding to $30 \ldots 60 \mathrm{~kW}$ ), could be represented by six $10-\mathrm{kW}$ virtual units with zero switching cost yielding binary integer problem. However, it has to be somehow taken into account that powers greater than 0 but less than $30 \mathrm{~kW}$ cannot be realized.

Three numerical optimization algorithms were implemented in Matlab: the first one based on simulated annealing (SA) [12], and the second one on genetic algorithms (GA) [12]. The third one was a simple steepest ascent algorithm. The simulated annealing technique was originally inspired by metallurgy. The process imitates the slow cooling of a solid. In the beginning the "temperature" of the optimized system is great thus allowing transitions to high-energy states (states that are far from optimal). This enables movement in the whole search space. As the system cools down, such transitions where the original state is less optimal than the new state become less and less likely. In the end, the system will "freeze" to a state that is a local optimum. The slower the cooling, the more likely it is that this is the global optimum. Genetic algorithms, on the other hand, derive their inspiration from the evolution of species and natural selection. An initial population of candidate solutions is formed, and successive generations are formed through recombination and mutations.

The SA-based optimizer was realized with the frequency converter-driven generator modeled as two 30-kilowatt virtual generators with zero switching costs. The algorithm required quite a lot of computational resources, and the results were still clearly sub-optimal (could easily be improved by a human analyzer).

In the GA-based algorithm, the converter-driven generator was modeled with a semi-continuous variable (from 0.60 to 1.20 with a step of 0.01). Two-point recombination was used, and the next generation was created using tournament selection. Two mutation mechanisms were implemented: replacing the generator utilization vector $\mathbf{e}$ with the vector of an adjacent hour, and replacing $\mathbf{e}$ with a random vector. The initial population was created randomly. The GA-based algorithm performed generally better than the SA-based one; the resulting income was greater, the computation time was smaller (minutes vs. hours) and the converterdriven generator could be more easily modeled in the GA-based optimizer than in the SA-based optimizer. Thus, the SA algorithm was dropped, and will not be further discussed in this article. 
The steepest ascent hill climbing algorithm first calculates the generator combination that produces the most constant head such that the e vector for each hour is the same. The converter-driven generator is modeled in the same way as in the GA-based optimizer. Then the algorithm tabulates all 24-hour long sequences that differ from the current one by a smallest possible change of the e vectors of one or two hours. The best of these is selected as the new solution. The process is repeated until no better solutions are found. Since the algorithm starts from the constant-head situation, it is likely to find a solution that is better than the constant-head utilization.

\section{Results}

The genetic algorithm-based and the steepest ascentbased optimization algorithms were tested with different price data and inflow values. In Fig. 3, an example of optimized generator combination sequence, acquired with the GA optimizer, is shown. The price profile is somewhat unusual, with a high peak during morning hours. The incoming flow was set to $50 \%$ of the combined nominal flow of the generators. As can be seen, the pool is filled to the maximum before the peaks, and the head is allowed to drop when the price is high. In this case, the generated revenue during the sequence was 97.03 euros, and 104.77 euros taking into account the end value and the penalties. The corresponding values for constant head control are 91.25 and 100.35 euros (head kept at initial). If the profit is, e.g., $10 \%$ of the daily income, the utilization of the optimization algorithm would increase the daily profit in this case by over $40 \%$ compared to constant head control. However, it should be noted that the price profile represents a very exceptional day.
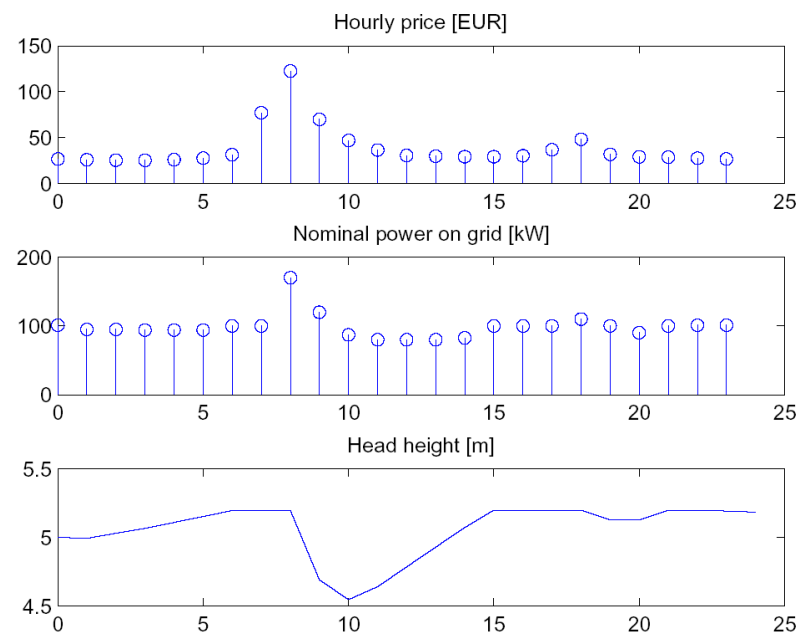

Fig. 3. An example of optimized generator combination sequence. The highest graph shows the hourly energy price (Eur/MWh), the middle graph is the utilized power (the output of the optimizer), and the lowest graph is the head (the upper water level). Sequence acquired using GA.
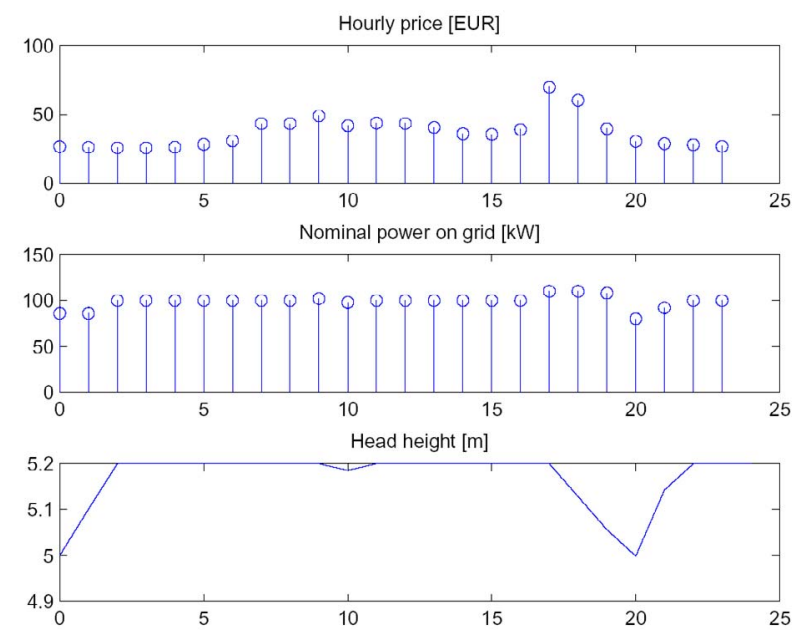

Fig. 4. An example of optimized generator combination sequence acquired with the steepest ascent method. The required controlling of the power is done exclusively using the converter-driven generator.

In Fig. 4 is presented another example of the optimization. The sequence was obtained using the steepest ascent method, and the inflow was the same as in the previous figure $(50 \%)$. The generated revenue was 92.00 euros (102.77 with penalties and remaining value). The corresponding values for constant-head control are 88.94 and 98.23 euros. In both cases (Figures 3 and 4) it should be noted that the constant-head control used as a reference could easily be improved by letting the pool fill to the maximum during the first hours and then keeping it at the maximum. In both cases, this yields a greater income than the basic constant head control but less than the optimized sequence (93.52/104.08 for Fig. 3 and 91.12/101.89 for Fig. 4). This maximum head strategy can be viewed as a special case of optimized utilization sequence, and its feasibility relative to constant head strategy will vary according to price profile and inflow. Thus the basic constant head strategy is better suited as a reference for optimized sequences.

The simulations were run for a few different price data sets and for a few different values of inflow. However, the number of simulations done thus far is insufficient for drawing general conclusions. The following observations were made, however:

- The steepest ascent hill climbing (SAHC) algorithm works, despite its simplicity, well. In all simulations calculated, it was able to reach a better utilization sequence than the constant head-control or the maximum-head control.

- The GA optimizer performs usually worse than SAHC, and is always more demanding in terms of computation time. However, sometimes it can produce better results than SAHC.

- The improvement of revenue (including value of the pool and penalties due to off-switchings) is approximately $0.5-10 \%$ compared to constant head control. Typical values lie between 1 and $4 \%$. If the profit is ca. $10 \%$ of the revenue, this translates to an increase of up to tens of percent 
in profit.

It should also be noted that the utilization of a frequency converter-driven generator is essential for price-based optimization of generator utilization. The overall economic efficiency of a small-scale head-dependent hydroelectric plant is more thoroughly discussed in [11].

\section{Conclusions and Further Work}

This paper presented methods to optimize the production of a small-scale head-dependent hydroelectric power station based on the electricity market price. A simple model for the power station with directly grid-connected and frequency converter-driven generators was presented. The optimization was realized in three versions: 1) Simulated Annealing (SA), 2) Genetic Algorithm (GA), and 3) Steepest Ascent Hill Climbing (SAHC). Simulated annealing proved quite ineffective in solving the problem. Genetic algorithm performed better, but had problems with some price profiles. SAHC is the simplest method in terms of computing capacity requirements, yet yields generally the best results. The optimization of the generator utilization improves the generated revenue by approximately $0.5-10 \%$ (taking into account value of the stored water and penalties arising from the wear of generator switches due to off-switching). Since the profit margin is typically small, probably less than $10 \%$, this translates to a profit increase of tens of percent.

The number of simulations was, however, insufficient for drawing generalized results. Therefore, more simulations are needed, especially a long-term simulation of the feasibility of the strategy. Simulations spanning at least a year - preferably several years - would be required to be able to state the increase in the profit. This will be done in the future. Furthermore, the system will be implemented in the pilot plant at Tirva to verify the simulations and gain experience of the requirements of the implementation of the actual system. The simulation model needs some improvements. Specifically, the relationship between the flow of the generators and the head, as well as the efficiency of the converter-driven turbine as a function of rotation speed should be added. Also the optimization methods could be improved, and new ones (e.g. tabu search) tested.

\section{References}

[1] O. Gjerde, "The Deregulated Nordic Electricity Market - 10 Years of Experience," Proceedings of the Transmission and Distribution Conference and Exhibition, Vol. 2. Yokohama, Japan, 6-10 Oct. 2002.

[2] O. B. Fosso, A. Gjelsvik, A. Haugstad, B. Mo, I. Wangensteen, "Generation scheduling in a deregulated system. The Norwegian case", IEEE Trans. on Power Systems, Vol. 14, No. 1, pp. 75-81, February 1999

[3] S. Kärkkäinen, E. Lakervi, "Liberalization of Electricity Market in Finland as a Part of Nordic
Market," in Generation, Transmission, and Distribution, IEE Proceedings. Vol. 148, Issue 2, pp. 194-199, March 2001.

[4] Nord Pool Spot WWW Page, http://www.nordpoolspot.com. Accessed Sep 15 2007.

[5] O. B. Fosso, M. M. Belsnes, "Short-term Hydro Scheduling in a Liberalized Power System", in the Proc. of 2004 Intl. Conf. on Power System Technology (POWERCON 2004), Singapore, Nov. 2004

[6] A. J. Conejo, J. M. Arroyo, J. Contreras, F. A. Villamor, "Self-Scheduling of a Hydro Producer in a Pool-Based Electricity Market", IEEE Trans. on Power Systems, Vol. 17, No. 4, pp. 1265-1272, Nov. 2002

[7] V. M. F. Mendez, C. Camus, J. P. S. Catalão, S. J. P. S. Mariano, L. A. F. M. Ferreira, "Educations on scheduling head-dependent hydro plants under competitive energy market." Proceedings of the 16th EAEEIE Annual Conference on Innovation in Education for Electrical and Information Engineering (EAEEIE'2005), Lappeenranta, Finland, 6-8 June, 2005.

[8] F. J. Nogales, J. Contreras, A. J. Conejo, R. Espínola, "Forecasting Next-Day Electricity Prices by Time Series Models", IEEE Trans. on Power Systems, Vol. 17, No. 2, pp. 342-348, May 2002

[9] C. P. Rodriguez, G. J. Anders, "Energy Price Forecasting in the Ontario Competitive Power System Market", IEEE Trans. on Power Systems, Vol. 19, No. 1, pp. 366-374, Feb. 2004

[10] A. J. Conejo, M. A. Plazas, R. Espínola, A. B. Molina, "Day-Ahead Electricity Price Forecasting Using the Wavelet Transform and ARIMA Models", IEEE Trans. on Power Systems, Vol. 20, No. 2, pp. 1035-1042, May 2005

[11] T. Lindh, R. Tiainen, J. Ahola, M. Niemelä, V. Särkimäki, "Performance of Rotational Speed Controlled Small-Scale Head-Dependent Hydroelectric Power Plant", to be published in the Proceedings of the Intl. Conf. on Renewable Energies and Power Quality 2008 (ICREPQ'08), Santander, Spain, March 2008

[12] S. Kirkpatrick and C. D. Gelatt and M. P. Vecchi, "Optimization by Simulated Annealing", Science, Vol 220, Number 4598, pages 671-680, 1983.

[13] J. M. Holland, Adaptation in Natural and Artificial Systems, MIT Press, $6^{\text {th }}$ Edition, 2001. ISBN 0-26258111-6 